\section{ITC $3 / 48$}

Journal of Information Technology and Control

Vol. 48/ No. 3/ 2019

pp. 401-414

DOI 10.5755/j01.itc.48.3.20470

\section{Dynamical Systems: Application to Francis Hydro-Turbine} Governing System

\title{
Stabilization of Arbitrary Switched Nonlinear Fractional Order Dynamical Systems: Application to Francis Hydro-Turbine Governing System
}

\section{Mona Faraji Niri}

Warwick Manufacturing Group, University of Warwick, Coventry CV4 7AL, United Kingdom; e-mail: Mona.Faraji-Niri@warwick.ac.uk

\section{Vahid Asadzadeh, Javad Rahmani Fard}

Electrical Engineering Department, Pooyesh Institute of Higher Education, Qom 3713956331, Iran; e-mails:Vahid.Asadzadeh@pooyesh.ac.ir, Javad.rahmani.fard@gmail.com

Corresponding author: Mona.Faraji-Niri@warwick.ac.uk

This paper is a theoretical and practical study on the stabilization of fractional order Lipschitz nonlinear systems under arbitrary switching. The investigated system is a generalization of both switched and fractional order dynamical systems. Firstly, a switched frequency distributed model is introduced as an equivalent for the system. Subsequently, a sufficient condition is obtained for the stabilizability of the system based on the Lyapunov approach. Finally, the results are extended to synthesis mode-dependent state feedback controller for the system. All the results are expressed in terms of coupled linear matrix inequalities, which are solvable by optimization tools and directly reducible to the conditions of the integer order nonlinear switching systems as well as the conventional non-switched nonlinear fractional order systems. The proposed method has various practical implications. As an example, it is utilized to control Francis hydro-turbine governing system. This system is represented as a switching structure and supposed to supply a load suffering abrupt changes driven by an arbitrary switching mechanism. The simulation results support the usefulness of the method.

KEYWORDS: Fractional Order System, Switched Dynamical System, Lyapunov Theory, Stabilization, Linear Matrix Inequality, Hydro-Turbine Governing System. 


\section{Introduction}

Switching systems are a class of hybrid systems that has attracted increasing attentions during the past decades. Various practical structures such as mechanical systems, power systems, networked controlled systems and multi agent systems could be described by switching dynamical systems [27]. This is due to the nature of switching systems in expressing the interactions between the continuous variable dynamics and the discrete events through multiple subsystems governed by a switching mechanism. These systems help to model the structural variations induced by external or internal discrete events such as failures, environmental factors, and configuration conversions [37, 38].

The stability, stabilization and control of switched systems are fundamental and interested research problems since they cannot be directly deduced from the specifications of each individual subsystem. A wide range of constructive researches is now available on the stability [19, 38, 41], stabilization and control [16-18], estimation and filtering [14, 47] of switched systems. In this area, the problems of nonlinear switched systems constitute a more significant focus since most real systems are essentially nonlinear but complex and hardly handled. Problems of nonlinear switched systems are addressed in $[33,38,45,49]$ and the references therein. Typically, the approach adopted to analyse these systems is utilizing the theories developed for nonlinear differential equations as well as the Lyapunov stability theory.

In all of the previous studies dedicated to the switched dynamical systems, the subsystems are supposed to be represented by the conventional integer order differential equations. It is already known that fractional calculus, as a generalization to the classical integer order calculus, provides a much better understanding of the realistic applications such as in electronic circuits $[1,21]$, electrical machines [35] and chemical systems [20]. Fundamentals of fractional order systems (FOSs) are pretty well established by now [4, 8, 31]. Various subtle results on the stability problems are discussed in $[10,26,39]$. Controllers are designed in $[23,24,29]$ and observer-based designs are proposed in [2, 11]. Further reviews are also reported in [31, 25].

An increased number of applications of FOSs in various areas of science and technology as well as the potentials of switching systems to model structure-varying systems necessitates the analysis of fractional order switching systems (FOSSs). There exist only few studies dedicated to FOSSs. Basic stability notions of FOSS are studied in [22, 46]. Stabilization and control problems of linear FOSSs are mentioned in $[3,5,6,29]$. Also, special classes of fractional order positive switched systems and fractional order impulsive switched system are studied in $[28,48]$ and $[13,44,48]$, respectively.

Despite the recent developments on switching systems and FOSs, the control problems of fractional order switched dynamical structures have not received enough attention. These systems have different features compared with ordinary integer order systems and studying their problems is more challenging than both integer order and switched systems. Accordingly, the contribution of this paper is to investigate the stabilizability and stabilization of such systems.

The main contribution of this paper is to study the stabilizability and controller design of a class of nonlinear continuous-time dynamical systems under arbitrary switching. The system under consideration is supposed to include a linear nominal part with an unknown nonlinearity of Lipschitz type. This class is interested since it can describe real physical systems more precise and direct than the conventional linear, non-switched or integer order systems. The problem is solved through introducing an equivalent switched frequency distributed model for the system. Based on the equivalent model, the stabilizability of the system is investigated based on the stability definitions of switched systems and fractional order systems. The result is the stabilizability condition obtained by the Lyapunov approach, as well as the stabilizing controller gains designed in terms of linear matrix inequalities (LMIs). The results obtained for FOSSs in this paper are reducible to the linear fractional order switching systems, the integer order switching systems and the conventional fractional order systems reported in the previous studies. As an example, the method is tested on Francis hydro-turbine governing power system with fractional order dynamics subject to nonlinearities and time-varying load profile which is modelled by a switched structure. The results support the usefulness of the method. 
The remainder of the paper is organized as follows: in Section 2, the dynamical specifications of the FOSSs are formulated and some preliminaries are recalled for both switching and fractional order systems. In Section 3, a stabilizability condition is derived and the controller is synthesized. In Section 4, the theoretical results are tested on the practical system. Finally, concluding remarks and possible future study directions are mentioned in Section 5.

\section{Preliminaries and Problem Formulation}

The fractional order nonlinear switched system (FONSS) is described as follows:

$$
\left\{\begin{aligned}
D^{\alpha} x(t) & =\mathbb{F}\left(x(t), u(t), r_{t}\right) \\
& =A\left(r_{t}\right) x(t)+f\left(x(t), r_{t}\right)+B\left(r_{t}\right) u(t)
\end{aligned}\right.
$$

where $x(t) \in \mathbb{R}^{n}$ is the state vector of the system, $u(t)$ $\in \mathbb{R}^{m}$ is the input vector of the system. $\left\{r_{t}, t \geq 0\right\}$ is a continuous-time switching mechanism [15] taking values in the finite set $\mathrm{N}=\{1,2, \ldots, N\}$, where the set $\mathrm{N}$ contains the modes of the system and $N$ is their number. The switching mechanism is of arbitrary type with no specific limitations or constraints.

$f\left(x(t), r_{t}\right)$ is a nonlinear dynamic related to $x(t)$ and $r_{t}$. $A\left(r_{t}\right)$ is the mode-dependent system matrix with compatible dimensions representing the nominal part of the system and $B\left(r_{t}\right)$ is a mode dependent system gain on the input $u(t)$. Also, $x_{0}$ is the initial state vector and $r_{0}$ represents the initial mode.

$D^{\alpha}$ denotes the fractional order operator representing both differential and integral operations. This operator is expressed by (2) according to the Caputo fractional derivative of the $\alpha$ th $(\alpha>0)$ order [9],

$$
D^{\alpha} h(t)=\frac{1}{\Gamma(n-\alpha)} \int_{0}^{t} \frac{h^{(n)}(\tau) d \tau}{(t-\tau)^{\alpha+1-n}},
$$

where $n$ is the nearest integer bigger than $\alpha$, i.e. $n$ is an integer satisfying $n-1<\alpha \leq n$. $h^{(n)}($.$) is the n$th derivative of the function $h($.$) and \Gamma$ (.) is the Gamma function defined by $\Gamma(z)=\int_{0}^{t} e^{-z} t^{z-1} d t$. Remarkably, $0<\alpha<1$ is the fractional commensurate order of the system.

Remark 1: Hereafter in the paper, for the convenience of notations, $r_{\mathrm{t}}=i$ is used and the subsystem matrices are labeled by $A_{i}, B_{i}, f_{i}(x(t))$ and $\mathbb{F}_{i}(x(t), u(t))$.

Remark 2: The FONSS of (1) is a general representation that can be used to model many physical systems in real world applications. The fractional differential equations enhance the representation precision while, the nominal part of (1) describes the linearization of the systems. Also, the nonlinear term represents various errors and uncertainties such as linearization errors or the external disturbances. Furthermore, the signal of $r_{t}$ justifies multiple operating points of the system each described by a distinct subsystem.

Assumption 1: The vector field of the system (1), $\mathbb{R}^{n} \times \mathbb{R}^{m} \rightarrow \mathbb{R}^{n}$ is continuous and differentiable at the origin; and therefore $\mathbb{F}_{i}(0,0)=0$.

Assumption 2: $f\left(x(t), r_{t}\right)$ is a continuous nonlinear function which is Lipschitz in $x$ with the Lipschitz constant $\gamma_{i}>0$, i.e.,

$$
\left\|f_{i}(\hat{x}(t))-f_{i}(x(t))\right\| \leq \gamma_{i}\|\hat{x}(t)-x(t)\|
$$

for all $\hat{x}(t), x(t) \in \mathbf{R}^{n}$ and $f_{i}(0)=0$.

Before proceeding further, the following lemmas, definition and proposition, which will be used in the derivation of the main results, are recalled.

Lemma 1: (Continuous frequency distributed model) [40]. The fractional order nonlinear differential equation $D^{\alpha} x(t)=g(x(t)$ can be expressed as (4) due to the continuous frequency distribute model of the fractional integrator,

$$
\left\{\begin{array}{l}
\frac{\partial Z(\omega, t)}{\partial t}=-\omega Z(\omega, t)+g(x(t)) \\
x(t)=\int_{0}^{\infty} \mu(\omega) Z(\omega, t) d \omega,
\end{array}\right.
$$

where $\mathrm{Z}(\omega, t)$ is an auxiliary time and frequency domain function and $\mu(\omega)$ is introduced as

$$
\mu(\omega)=\frac{\sin (\alpha \pi)}{\pi} \omega^{-\alpha} .
$$

Lemma 2: (Schur complement) [7]. For a given ma- 
$\operatorname{trix} S=S^{T}$, the following assertions are equivalent:

$$
\begin{aligned}
& S=\left[\begin{array}{ll}
S_{11} & S_{12} \\
S_{21} & S_{22}
\end{array}\right]<0 \\
& S_{11}<0, S_{22}-S_{12}^{T} S_{11}^{-1} S_{12}<0 \\
& S_{22}<0, S_{11}-S_{12}^{T} S_{22}^{-1} S_{12}<0 .
\end{aligned}
$$

Lemma 3: [42] Let $Y$ be a symmetric matrix, $H$ and $E$ be given matrices with the appropriate dimensions. For $F(t)$ satisfying $F(t)^{T} F(t) \leq I$, the inequality (9) holds

$$
H F E+E^{T} F^{T} H^{T}<0
$$

if and only if there exists an $\varepsilon>0$ such that

$$
H F E+E^{T} F^{T} H^{T} \leq \varepsilon H H^{T}+\varepsilon^{-1} E^{T} E .
$$

Definition 1: (Common Lyapunov Function) [38]. A function $V(x(t))$ is said to be a common (strong) Lyapunov function for the conventional switching system $\dot{x}(t)=g\left(x(t), r_{t}\right)$ if:

1 It is continuous everywhere and continuously differentiable except possibly at the origin.

2 It admits class $K_{\infty}$ bounds, i.e. there are class $K_{\infty}$ functions of $\beta_{1}$ and $\beta_{2}$ such that, $\beta_{1}(|x(t)|) \leq V(x(t))$ $\leq \beta_{2}(|x(t)|)$.

3 There is a class $K$ function $\beta_{3}$ such that $\dot{V}(x(t)) \leq$ $-\beta_{3}(|x(t)|)$.

Proposition 1: (Stability) [38]. The switched system $\dot{x}(t)=g\left(x(t), r_{t}\right)$ is uniformly asymptotically stable if it admits a common Lyapunov function.

By introducing the FONSSs as a generalization of both switching and fractional order nonlinear systems, now, the stabilizability and stabilization problems can be addressed.

\section{Main Results}

In this section, the stabilization problem of nonlinear fractional order switched dynamical system is investigated. To this end, first, the continuous mode-dependent frequency distributed model is obtained for the system. Subsequently, Lyapunov approach is utilized to give the condition. All the results are reported in the LMI form and can be checked easily.

Consider the state feedback controller of the following form:

$u(t)=K\left(r_{t}\right) x(t)$

where $K\left(r_{t}\right)$ is the mode dependent gain to be designed.

Applying the controller gains of (11) to the system of (1) yields the closed-loop system as (12),

$$
\left\{\begin{array}{l}
D^{\alpha} x(t)=\bar{A}\left(r_{t}\right) x(t)+f\left(x(t), r_{t}\right) \\
x\left(t_{0}\right)=x_{0}, r_{t_{0}}=r_{0},
\end{array}\right.
$$

where

$$
\bar{A}\left(r_{t}\right)=A\left(r_{t}\right)+B\left(r_{t}\right) K\left(r_{t}\right) .
$$

By using the Lyapunov approach, first the sufficient condition for stability of the closed-loop dynamic system is obtained.

Theorem: The FONSS of (1) with the commensurate order $\alpha, 0<\alpha<1$ is stabilizable with the controller gains (11) if, there are symmetric, positive definite matrix $X$, matrices $Y_{i}$ together with the real scalars $\varepsilon_{i}>0$ and $\gamma_{i}>0$ such that the following set of LMIs hold:

$$
\left[\begin{array}{cc}
J_{i} & \gamma_{i} X \\
\gamma_{i} X & \varepsilon_{i} I
\end{array}\right]<0
$$

where

$$
J_{i}=X A_{i}^{T}+X A_{i}+Y_{i}^{T} B_{i}^{T}+B_{i} Y_{i}+\varepsilon_{i} I .
$$

Then, the controller gains are obtained as $K_{i}=Y_{i} X^{-1}$.

Proof: Following Lemma 1, the closed-loop dynamic system of (12) can be written as:

$$
\left\{\begin{array}{l}
\frac{\partial Z(\omega, t)}{\partial t}=-\omega Z(\omega, t)+\bar{A}\left(r_{t}\right) x(t)+f\left(x(t), r_{t}\right) \\
x(t)=\int_{0}^{\infty} \mu(\omega) Z(\omega, t) d \omega .
\end{array}\right.
$$

Based on the continuous frequency distributed system (16), consider the two Lyapunov functions: 
$v(\omega, t)$ as the Lyapunov function corresponding to the elementary frequency $\omega$ and $V(t)$ defined by (17) as the Lyapunov function summing all the $v(\omega, t)$ with the weighting function $\mu(\omega)$. Here, $P$ denounces a symmetric and positive definite matrix.

$V(t)=\int_{0}^{\infty} \mu(\omega) v(\omega, t) d \omega=\int_{0}^{\infty} \mu(\omega) Z^{T}(\omega, t) P Z(\omega, t) d \omega$.

The derivative of the Lyapunov function along the solution trajectories of (16) becomes as (18)

$$
\begin{aligned}
& \dot{V}(Z(t))=\int_{0}^{\infty} \mu(\omega) \dot{Z}^{T}(\omega, t) P Z(\omega, t) d \omega+ \\
& \int_{0}^{\infty} \mu(\omega) Z^{T}(\omega, t) P \dot{Z}(\omega, t) d \omega .
\end{aligned}
$$

Substituting the equations of (16) into (18) provides:

$\dot{V}(Z(t))=\int_{0}^{\infty}\left(\mu(\omega)\left\{-\omega Z(\omega, t)+\bar{A}_{i} x(t)+f_{i}(x(t))\right\}^{T} P Z(\omega, t)\right) d \omega+$ $\int_{0}^{\infty}\left(\mu(\omega) Z^{T}(\omega, t) P\left\{-\omega Z(\omega, t)+\bar{A}_{i} x(t)+f_{i}(x(t))\right\}\right) d \omega$.

Simplifications yield:

$$
\begin{aligned}
& \dot{V}(Z(t))=-2 \int_{0}^{\infty} \omega \mu(\omega) Z^{T}(\omega, t) P Z(\omega, t) d \omega+ \\
& \int_{0}^{\infty} \mu(\omega) x^{T}(t) \bar{A}_{i}^{T} P Z(\omega, t) d \omega+ \\
& \int_{0}^{\infty} \mu(\omega) Z^{T}(\omega, t) P \bar{A}_{i} x(t) d \omega+ \\
& \int_{0}^{\infty} \mu(\omega) f_{i}^{T}(x(t)) P Z(\omega, t) d \omega+ \\
& \int_{0}^{\infty} \mu(\omega) Z^{T}(\omega, t) P f_{i}(x(t)) d \omega
\end{aligned}
$$

which turns to the following using (16),

$$
\begin{aligned}
& \dot{V}(Z(t))=-2 \int_{0}^{\infty} \omega \mu(\omega) Z^{T}(\omega, t) P Z(\omega, t) d \omega+ \\
& x^{T}(t) \bar{A}_{i}^{T} P x(t)+x^{T}(t) P \bar{A}_{i} x(t)+ \\
& f_{i}^{T}(x(t)) \operatorname{Px}(t)+x^{T}(\omega, t) P f_{i}(x(t))
\end{aligned}
$$

The first item in the right-hand side of (21) is less than zero, therefore the inequality (22) holds

$$
\begin{aligned}
& -2 \int_{0}^{\infty} \omega \mu(\omega) Z^{T}(\omega, t) P Z(\omega, t) d \omega+x^{T}(t) \bar{A}_{i}^{T} P x(t)+ \\
& x^{T}(t) P \bar{A}_{i} x(t)+f_{i}^{T}(x(t)) P x(t)+x^{T}(t) P f_{i}(x(t)) \leq \\
& x^{T}(t) \bar{A}_{i}^{T} P x(t)+x^{T}(t) P \bar{A}_{i} x(t)+f_{i}^{T}(x(t)) P x(t)+ \\
& x^{T}(t) P f_{i}(x(t))
\end{aligned}
$$

Subsequently, if the inequality (23) holds for $\forall i \in \underline{N}$,

$$
\begin{aligned}
& x^{T}(t) \bar{A}_{i}^{T} P x(t)+x^{T}(t) P \bar{A}_{i} x(t)+f_{i}^{T}(x(t)) P x(t)+ \\
& x^{T}(t) P f_{i}(x(t))<0
\end{aligned}
$$

one has $\dot{V}(Z(t))<0$. Substituting (13) in (23) leads to (24)

$$
\begin{aligned}
& x^{T}(t)\left(A_{i}+B_{i} K_{i}\right)^{T} P x(t)+x^{T}(t) P\left(A_{i}+B_{i} K_{i}\right) x(t)+ \\
& f_{i}^{T}(x(t)) P x(t)+x^{T}(t) P f_{i}(x(t))<0 .
\end{aligned}
$$

Remarkably, (24) can be rewritten as (25) utilizing the following inequality obtained by Lemma 3

$$
\begin{aligned}
& f_{i}^{T}(x(t)) P x(t)+x^{T}(t) P f_{i}(x(t)) \leq \\
& \varepsilon_{i} x^{T}(t) P^{2} x(t)+\varepsilon_{i}^{-1} \gamma_{i}^{2} x^{T}(t) x(t) .
\end{aligned}
$$

Utilizing (25), the inequality of (24) turns to (26):

$$
\begin{aligned}
& x^{T}(t)\left(A_{i}+B_{i} K_{i}\right)^{T} P x(t)+x^{T}(t) P\left(A_{i}+B_{i} K_{i}\right) x(t)+ \\
& \varepsilon_{i} x^{T}(t) P^{2} x(t)+\gamma_{i}^{2} \varepsilon_{i}^{-1} x^{T}(t) x(t)<0
\end{aligned}
$$

which is equivalent to ( 27 ) due to its symmetric form:

$$
\left(A_{i}+B_{i} K_{i}\right)^{T} P+P\left(A_{i}+B_{i} K_{i}\right)+\varepsilon_{i} P^{2}+\varepsilon_{i}^{-1} \gamma_{i}^{2}<0
$$

Considering the quadratic and bounded Lyapunov function of (17) along with its negative definite form guaranteed by (27), the closed-loop system of (12) is stabilizable according to Definition 1 and Proposition 1.

Notably, the condition of (27) is nonlinear in $P$ and $K_{i}$. To find the controller gains, it is desired to transform (27) into an LMI form, so let $X=P^{-1}$. Pre- and post-multiplying (27) by $X$ yields (28). 
$X A_{i}^{T}+X A_{i}+X K_{i}^{T} B_{i}^{T}+B_{i} K_{i} X+\varepsilon_{i} I+\varepsilon_{i}^{-1} \gamma_{i}^{2} X^{2}<0,(28)$

Define $Y_{i}=K_{i} X$. Therefore, (27) changes to (29):

$X A_{i}^{T}+X A_{i}+Y_{i}^{T} B_{i}^{T}+B_{i} Y_{i}+\varepsilon_{i} I+\varepsilon_{i}^{-1} \gamma_{i}^{2} X^{2}<0,(29)$

Using Lemma 2 makes it possible to write (29) in the form of (14) and (15). Finally, the state feedback gains are derived as $K_{i}=Y_{i} X^{-1}$, which ends the proof.

The flowchart of the proposed method is shown in Figure 1.

Figure 1

The flowchart of the proposed method

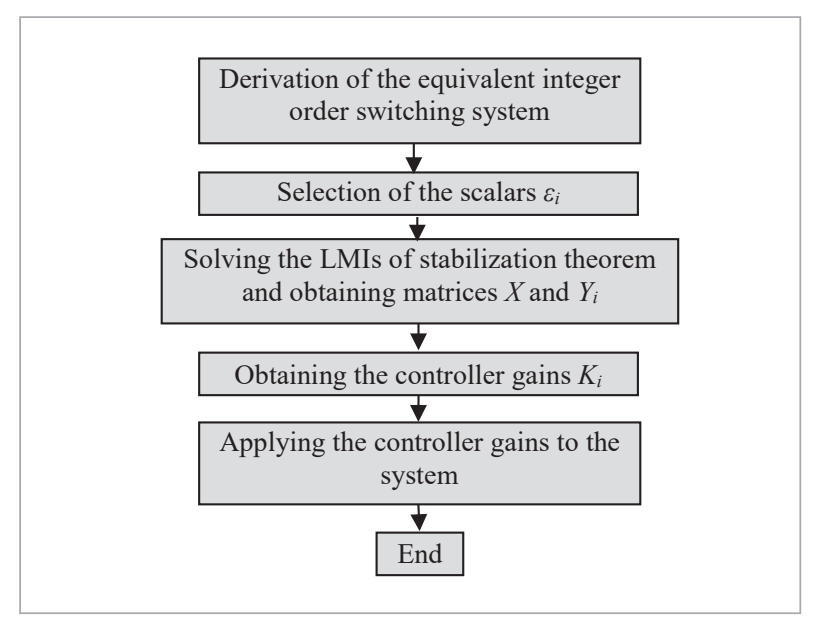

Remark 3: The stability criterion obtained for the nonlinear fractional order switched system is directly reducible to the results which have been previously reported for the integer order switched systems [38], and the ordinary fractional order systems $[32,36]$.

\section{Practical Application}

In this section, the simulation results are provided for a nonlinear fractional order hydro-turbine governing system (HGS) [43] to show the merits of the proposed method.

The fractional order Francis hydro-turbine governing system is composed of a hydro-turbine and penstock system, a generator system and a hydraulic servo sys- tem. The system is modelled as a switching structure which is supposed to supply a load that suffers abrupt changes driven by an arbitrary switching mechanism.

\subsection{Modelling of the Switched Nonlinear Francis Hydro-Turbine Governing System}

The basic physical model of the hydro power penstock system is shown in Figure 2.

\section{Figure 2}

Schematic of Francis hydro Turbine Governing System

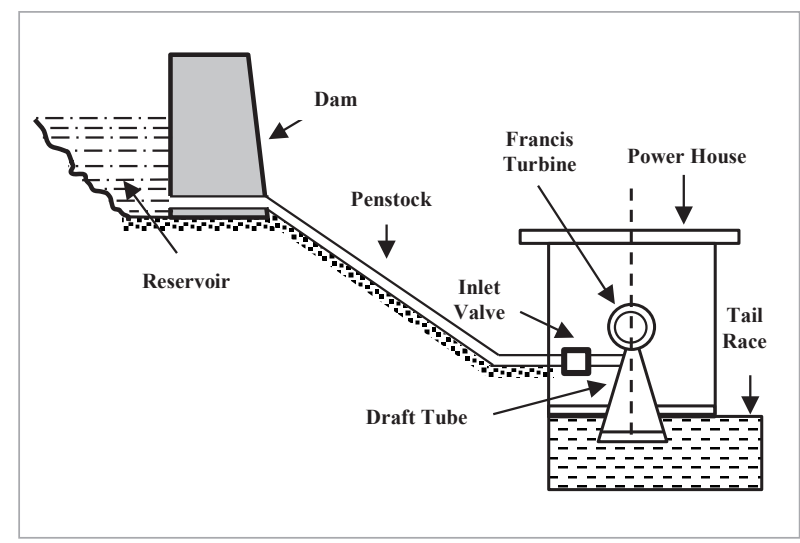

The dynamic characteristic of the synchronous generator is given by:

$$
\begin{aligned}
& \dot{\delta}(t)=\omega_{0} \omega(t) \\
& \dot{\omega}(t)=\frac{1}{T_{a}+T_{b}}\left(-D \omega(t)+m_{t}(t)-m_{e}(t)\right),
\end{aligned}
$$

where $\delta(t)$ is the rotor angle, $\omega(t)$ is the variation of the generator speed and $\omega_{0}$ is the rated angular speed of the generator. Additionally, $D$ is the damping factor of the generator and it is generally regarded as a constant. $m_{t}(t)$ is the output torque of the hydro turbine while $m_{e}(t)$ denotes the torque of the electrical load. $T_{a}$ is the inertia time constant of the generator and $T_{b}$ denotes the inertia time constant of the load.

If the influence of the rotor on the torque is added to the damping factor, the torque of the electric load and the terminal active power are equal to each other, i.e.

$$
P_{e}(t)=m_{e}(t)
$$

The electromagnetic power of the generator can be described as (32) 


$$
P_{e}(t)=\frac{E_{q}^{\prime} V_{s}}{x_{d}^{\prime}} \sin \delta(t)+\frac{V_{s}^{2}}{2} \frac{x_{d}^{\prime}-x_{q}}{x_{d}^{\prime} x_{q}} \sin 2 \delta(t),
$$

where $E_{q}^{\prime}$ is the transient internal voltage of the armature, $V_{s}$ is the voltage of bus at infinity, $x_{d}^{\prime}$ and $x_{q}$ are the direct axis and quartered transient reactances, respectively.

Dynamic characteristic of a hydraulic servo system is given as (33) according to the fractional calculus

$$
D^{\alpha} y(t)=\frac{1}{T_{y}}(u(t)-y(t)),
$$

where $y(t)$ is the incremental deviation of the guide vane opening and $T_{y}$ is the major relay connector response time. Here, $u(t)$ is the output signal of the governing system, which is the input voltage for the electric-hydraulic servo system.

The output torque of the turbine governing system is obtained as

$$
\dot{m}_{t}(t)=\frac{1}{e_{q h} T_{\omega}}\left(-m_{t}(t)+e_{y} y(t)+\frac{e e_{y} T_{\omega}}{T_{y}} y(t)\right) .
$$

In (34), $e_{q h}$ is the transfer coefficient of turbine flow on the head, $T_{\omega}$ is the water inertia time constant of the penstock system, $e_{y}$ is the transfer coefficient of turbine torque flow on the servo motor stroke, $e$ is defined as $e=e_{q h} e_{h} / e_{y}-e_{q h}$, and $e_{h}$ is the transfer coefficient of turbine torque on the water head.
It is supposed that the system load is varying according to an arbitrary switching mechanism.

The load is assumed to be characterized by two distinguished inertia time constants characterized by $T_{b}\left(r_{t}\right)$ :

$$
T_{b}\left(r_{t}\right)=\left\{\begin{array}{cc}
T_{b 1} & r_{t}=1 \\
T_{b 2} & r_{t}=2,
\end{array}\right.
$$

where $T_{b 1}$ refers to load with large time constant while $T_{b 2}$ specifies a load with small time constant.

The block diagram of the proposed control system is depicted in Figure 3. The system is composed of a switching controller whose modes change according to the same switching mechanism that orchestrates the loads.

Considering the Equations (30) to (35), the mathematical switching model of the hydro-turbine governor system is obtained as (36) where the fractional order is $\alpha=0.98$ :

$$
\left\{\begin{aligned}
\dot{\delta}(t) & =\omega_{0} \omega(t) \\
\dot{\omega}(t) & =\frac{1}{T_{a}+T_{b}\left(r_{t}\right)}\left(-D \omega(t)+m_{t}(t)-\frac{E_{q}^{\prime} V_{s}}{x_{d \Sigma}^{\prime}} \sin \delta(t)\right. \\
& \left.-\frac{V_{s}^{2}}{2} \frac{x_{d}^{\prime}-x_{q}}{x_{d}^{\prime} x_{q}} \sin 2 \delta(t)\right) \\
\dot{m}_{t}(t) & =\frac{1}{e_{q h} T_{\omega}}\left(-m_{t}(t)+e_{y} y(t)+\frac{e e_{y} T_{\omega}}{T_{y}} y(t)\right) \\
D^{\alpha} y(t) & =\frac{1}{T_{y}}(u(t)-y(t)) .
\end{aligned}\right.
$$

Figure 3

The proposed control structure for the Francis hydro-turbine governing system

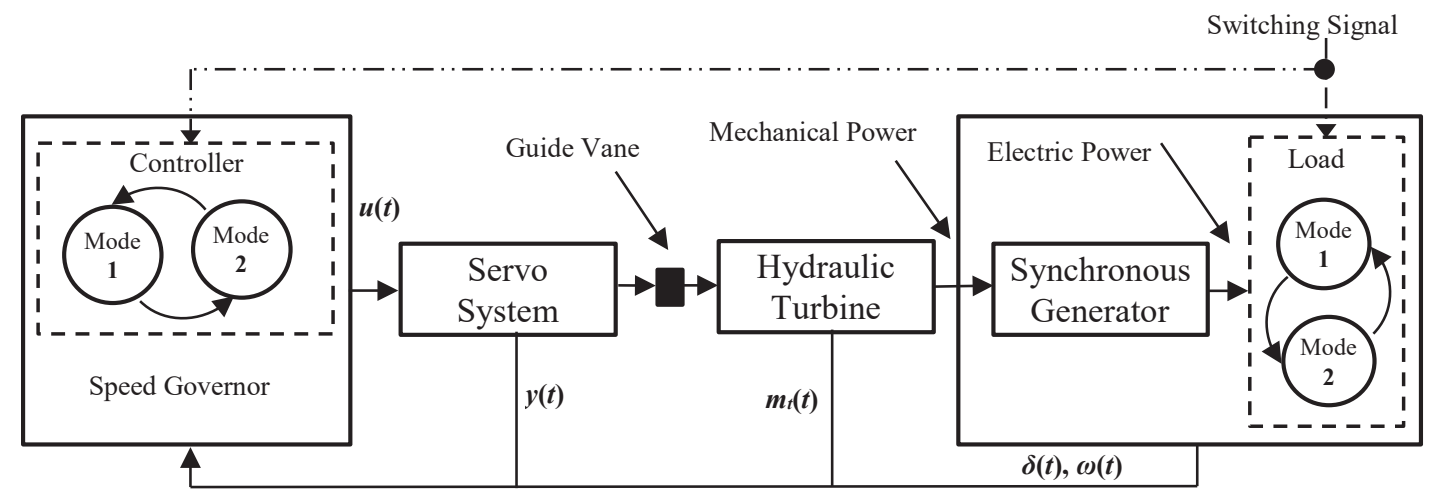


To proceed further, the parameters of system (35) are selected as shown in Table 1. Remarkably, the state variables as well as the parameters are in p.u. while the time constants are in seconds.

Table 1

Parameter values of the HGS system

\begin{tabular}{c|c|c|c} 
Parameter & Value & Parameter & Value \\
\hline$D$ & 2 & $\omega_{0}$ & 300 \\
\hline$T_{\omega}$ & 0.8 & $T_{y}$ & 0.1 \\
\hline$T_{a}$ & 10 & $T_{b 1}$ & 9 \\
\hline$T_{b 2}$ & 0.6 & $V_{s}$ & 1 \\
\hline$x_{q}$ & 1.474 & $x_{q}{ }^{\prime}$ & 1.25 \\
\hline$e$ & 0.7 & $e_{q h}$ & 0.5 \\
\hline$e_{y}$ & 1 & - & - \\
\hline
\end{tabular}

Defining the state variables of the HGS as $x_{1}(t)=\delta(t), x_{2}(t)=\omega(t), x_{3}(t)=m_{t}(t), x_{4}(t)=y(t)$ and replacing the parameter values, reveals the final form of the system model as (37)

$$
\left\{\begin{array}{l}
\dot{x}_{1}(t)=300 x_{2}(t) \\
\dot{x}_{2}(t)=\frac{1}{10+T_{b}\left(r_{t}\right)}\left(-2 x_{2}(t)+x_{3}(t)-1.08 \sin x_{1}(t)\right. \\
\left.\quad-0.061 \sin 2 x_{1}(t)\right) \\
\dot{x}_{3}(t)=-2.5 x_{3}(t)+6.6 x_{4}(t) \\
D^{0.98} x_{4}(t)=-10 x_{4}(t)+10 u(t)
\end{array}\right.
$$

Accordingly, the system matrices in the form of (1) are obtained as (38):

$$
\begin{aligned}
& A_{1}=\left[\begin{array}{cccc}
0 & 300 & 0 & 0 \\
0 & \frac{-2}{19} & \frac{1}{19} & 0 \\
0 & 0 & -2.5 & 6.6 \\
0 & 0 & 0 & -10
\end{array}\right], A_{2}=\left[\begin{array}{cccc}
0 & 300 & 0 & 0 \\
0 & \frac{-2}{10.6} & \frac{1}{10.6} & 0 \\
0 & 0 & -2.5 & 6.6 \\
0 & 0 & 0 & -10
\end{array}\right], \\
& B_{1}=B_{2}=\left[\begin{array}{llll}
0 & 0 & 0 & 10
\end{array}\right]^{T} \\
& f_{1}(x(t))=\left[\begin{array}{lll}
0 \frac{-1.08}{19} \sin x_{1}(t)+\frac{-0.061}{19} \sin 2 x_{1}(t) & 0 & 0
\end{array}\right]^{T} \\
& f_{2}(x(t))=\left[\begin{array}{llll}
0 & \frac{-1.08}{10.6} \sin x_{1}(t)+\frac{-0.061}{10.6} \sin 2 x_{1}(t) & 0 & 0
\end{array}\right]^{T} \text {. }
\end{aligned}
$$

\subsection{Simulation Results of the Switched Nonlinear Francis hydro-Turbine Governing System}

Uncontrolled states of the fractional order HGS under two different arbitrary switching signals shown by Figure 4 are illustrated in Figure 5. As the figure shows, the uncontrolled system is unstable.

\section{Figure 4}

Two sample arbitrary switching signals (SW1, SW2)

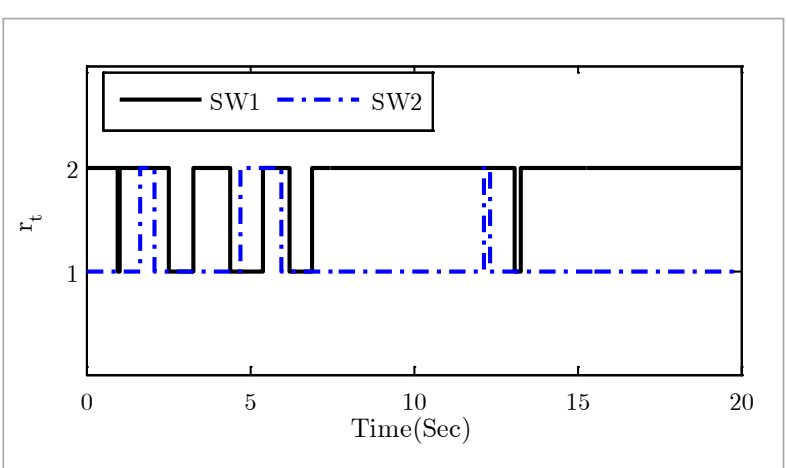

\section{Figure 5}

Uncontrolled states of HGS under two arbitrary switching signals (SW1, SW2)
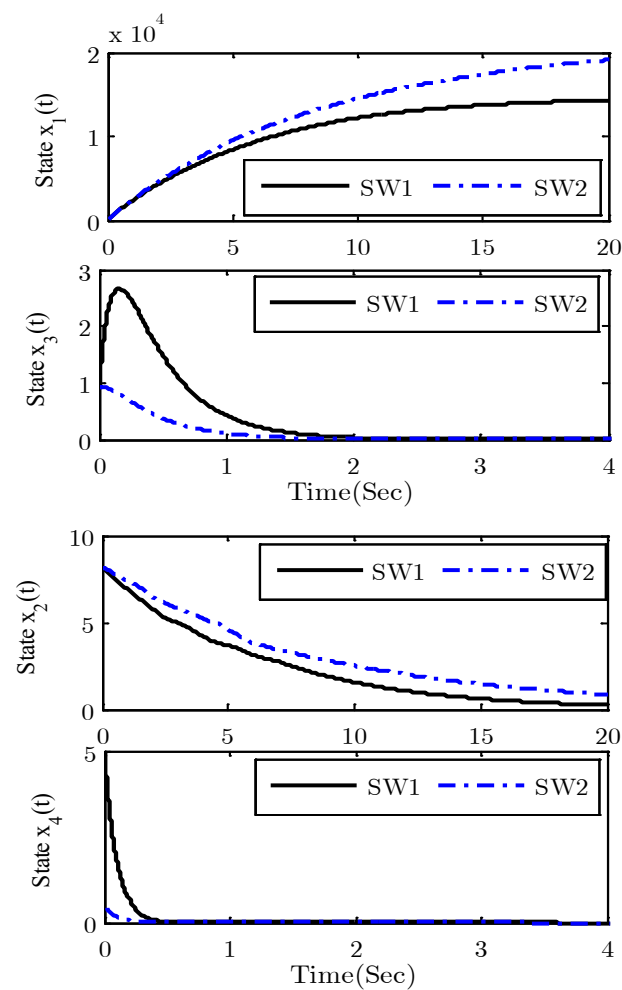
Solving the LMIs of Theorem provides the matrices and controller gains as (39). The parameters are selected as $\gamma_{1}=\gamma_{2}=10 \mathrm{e}-10, \varepsilon_{1}=\varepsilon_{1}=10 \mathrm{e}-\%$.

The resultant control outputs of the HGS appear in Figure 6. The controlled states of the HGS are shown in Figure 7. Clearly, the states are stabilized properly.

\section{Figure 6}

Controller outputs under two arbitrary switching signals

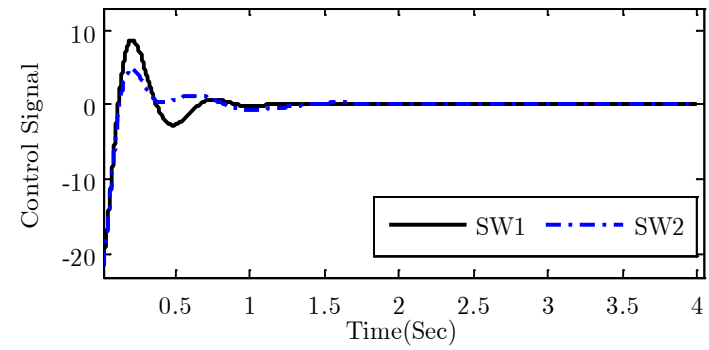

Figure 7

Controlled states of HGS under two arbitrary switching signals (SW)
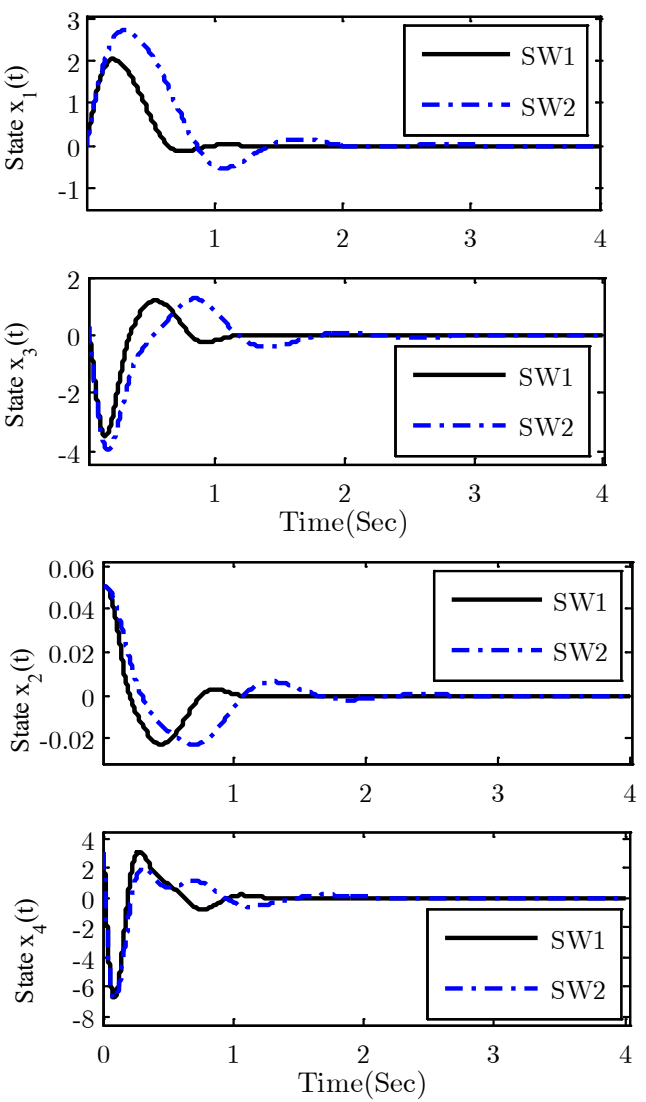

$X=\left[\begin{array}{cccc}0.2237 & -0.0017 & -0.0025 & 0.0421 \\ -0.0017 & -0.0003 & -0.0014 & -0.0011 \\ -0.0025 & -0.00145 & 0.1651 & -0.0198 \\ 0.0421 & -0.0011 & -0.0201 & 0.2504\end{array}\right]$

$\mathrm{Y}_{1}=\left[\begin{array}{llll}0.0611 & -0.0008 & -0.1839 & 0.1802\end{array}\right]$,

$\mathrm{Y}_{2}=\left[\begin{array}{llll}0.0548 & -0.0008 & -0.1808 & 0.1811\end{array}\right]$

$K_{1}=\left[\begin{array}{llll}-2.4054 & -364.723 & -4.4487 & -0.8520\end{array}\right]^{T}$,

$K_{2}=\left[\begin{array}{llll}-2.3584 & -353.8254 & -4.3276 & -0.8024\end{array}\right]^{T}$.

To further investigate the proposed theorem, the simulations are conducted fot 100 different switching signals. The statistics of the settling time and its approximated distribution are summarized in Figure 8. The figure shows that the convergence time has a normal distribution with mean value of 1.2217 seconds and the deviation of 0.2174 seconds.

\section{Figure 8}

Statistics of the settling time of the controlled states for 100 different arbitrary signals

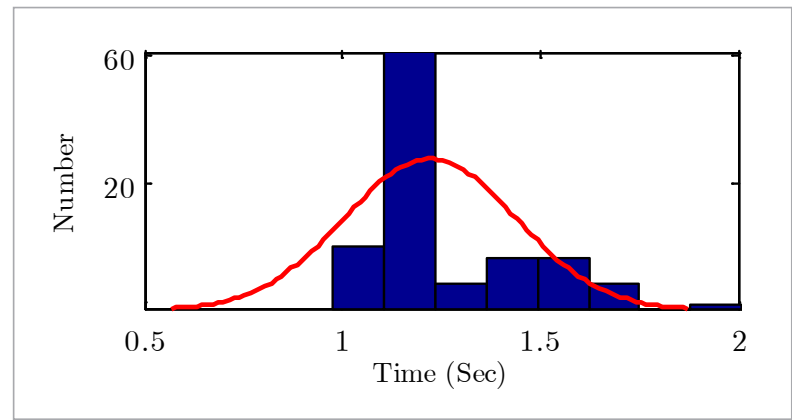

Remarkably, a drawback of the proposed method is that the conditions depend on a number of parameters $\varepsilon$ and $\gamma$ that must be suitably tuned. The parameter $\varepsilon$ rises due to Lemma 3 used for dealing with the system nonlinearity. According to [42], this lemma holds for any $\varepsilon>0$. Although this parameter could take any values, it is preferred to be selected properly. The reason is that this parameter determines the robustness degree of the system and improper values may increase conservativeness and even lead to infeasible LMI sets.

To investigate the effect of $\varepsilon$ on the responses, the controlled system states and control signals are depicted in Figures 9-10 under the switching of Figure 11. 
Figure 9

The effect of the parameter $\varepsilon$ on the controlled states of HGS
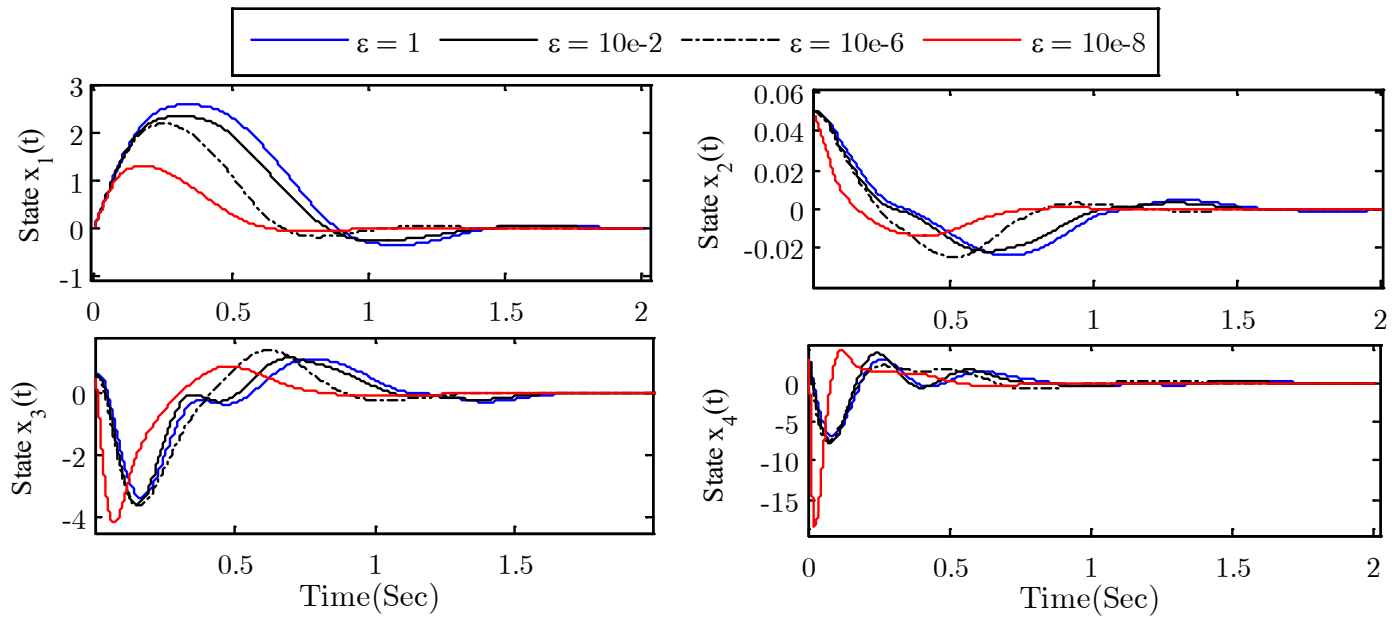

Figure 10

The effect of $\varepsilon$ on the control signal variation

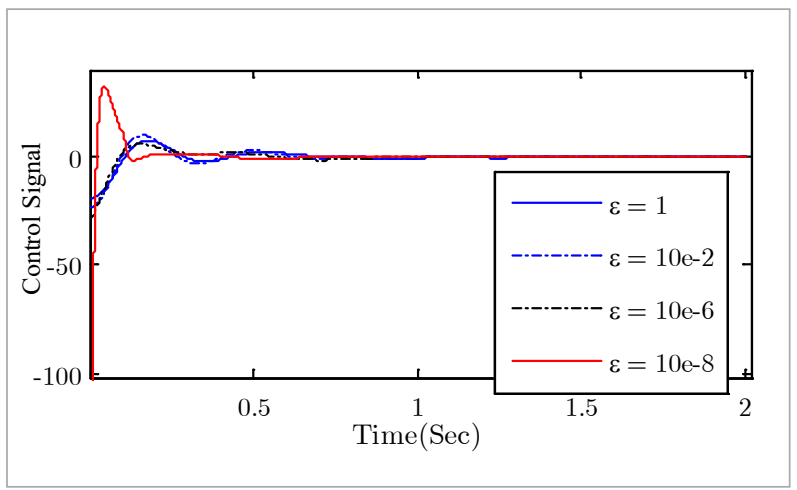

Figure 11

Sample arbitrary switching signals for $\varepsilon$ analysis for the load variation

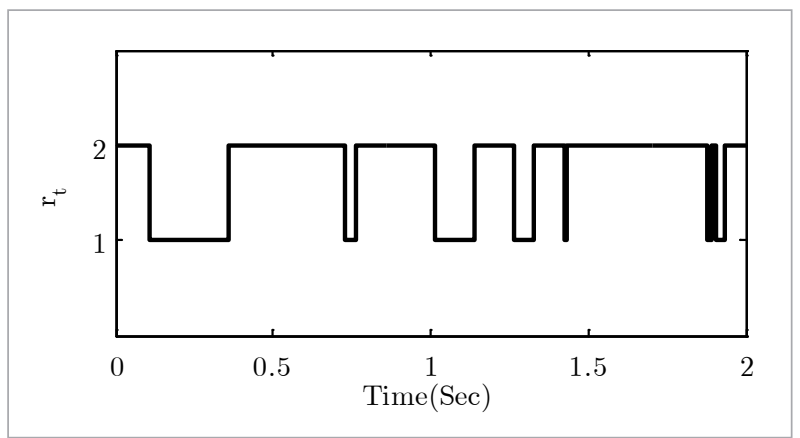

Similarly, the parameter of $\gamma$ appears as a result of Assumption 2 , it is a positive value that fulfils the Lipschitz condition. The effect of this parameter is also simulated in Figures 12-14.

As the figures show, smaller values of both parameters $\varepsilon$ and $\gamma$ lead to faster and less conservative responses.

At the same time, they provide more aggressive and larger controller outputs. Since the variations of the controller output and the conservativeness of the responses are conflicted specifications, proper selection of both parameters is required.

There exist two approaches for dealing with the parameters $\varepsilon$ and $\gamma$. The first approach is to select them a priori to afford a prescribed degree of robustness. This approach is extensively used in the controller design problems [44] and is also preferred in the current study. The main advantage of this approach is providing the conditions of a fair comparison between the multiple results. The second approach is to optimize those parameters which is addressed in [34]. It is worth mentioning that, for analysis, the parameters of $\varepsilon$ and $\gamma$ are selected the same for both modes without loss of generalization. Additionally, to provide a fair comparison between the results of distinct values of $\varepsilon$ and $\gamma$, for the former $\gamma$ is uniformly selected as $\gamma=10 \mathrm{e}-9$, while for the latter $\varepsilon$ is uniformly selected as $\varepsilon=10 \mathrm{e}-8$ for both modes. 
Figure 12

Sample arbitrary switching signals for $\gamma$ analysis for the load variation

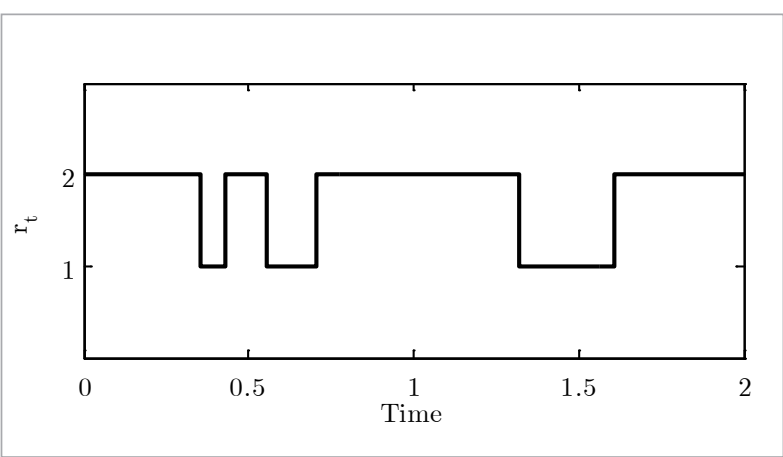

Figure 13

The effect of $\gamma$ on the control signal variation

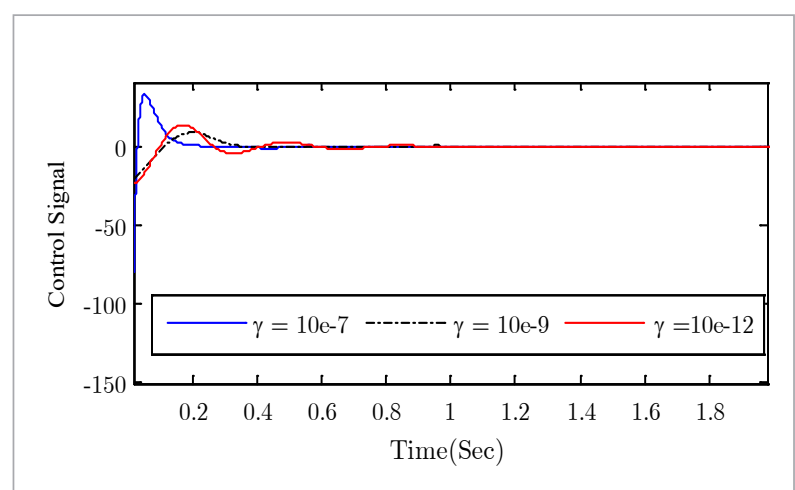

\section{Figure 14}

The effect of the parameter $\gamma$ on the controlled states of HGS
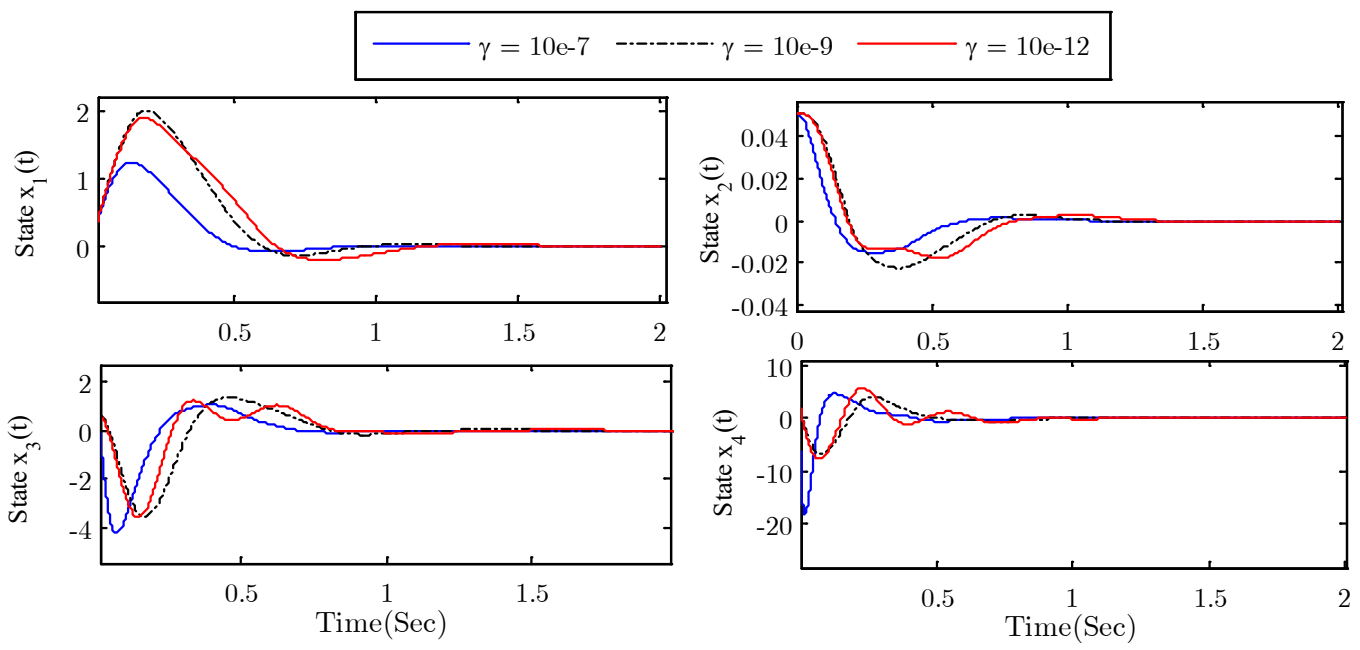

\section{Conclusions}

In this paper, the stabilizability problem of nonlinear fractional order systems under arbitrary switching is addressed. The analysis is started by means of a frequency distributed mode-dependent equivalent model for the system. Subsequently, a condition guaranteeing the existence and the synthesis of multi-mode feedback stabilizing controller in LMI formulation is given. The computed controller law ensures the stabilization of the system under the nonlinear perturbations and the dynamical variations induced by the switching. The obtained theorem is a general form of the results previously reported for non-switched fractional order systems as well as the ordinary integer order switched systems. The proposed analysis and synthesis method is successfully tested on a hydro-turbine governing system modelled as a switching structure. Remarkably, further studies are required on the nonlinear fractional order dynamical systems under constrained switching mechanisms and their applications. 


\section{References}

1. Aguila-Camacho, N., Le Roux, J. D., Duarte-Mermoud, M. A., Orchard, M. E. Control of a Grinding Mill Circuit Using Fractional Order Controllers. Journal of Process Control, 2017, 53, 80-94. https://doi.org/10.1016/j.jprocont.2017.02.012

2. Azami, A., Naghavi, S. V., Dadkhah Tehrani, R., Khooban, M. H., Shabaninia, F. State Estimation Strategy for Fractional Order Systems with Noises and Multiple Time Delayed Measurements. IET Science, Measurement \& Technology, 2017, 11(1), 9-17. https://doi. org/10.1049/iet-smt.2016.0089

3. Babiarz, A. Grzejszczak, T., Łęgowski A., Niezabitowski, M. Controllability of Discrete-Time Switched Fractional Order Systems. 12th World Congress on Intelligent Control and Automation, Guilin, China, June 2016, 1754-175\%. https://doi.org/10.1109/WCICA.2016.7578750

4. Baleanu, D., Machado, J. A. T., Luo A. C. J. (Eds.). Fractional Dynamics and Control. Springer Science and Business Media, 2011. https://doi.org/10.1007/978-14614-0457-6

5. Balochian, S. Stabilization of Autonomous Linear Time Invariant Fractional Order Derivative Switched Systems with Different Derivative in Subsystems. Bulletin of the Polish Academy of Sciences Technical Sciences, 2014, 62, 495-503. https://doi.org/10.2478/bpasts-2014-0053

6. Balochian, S., Khaki Sedigh, A. Sufficient Condition for Stabilization of Linear Time Invariant Fractional Order Switched Systems and Variable Structure Control Stabilizers. ISA Transactions, 2012, 51(1), 65-73. https:// doi.org/10.1016/j.isatra.2011.07.010

7. Boyd, S., El Ghaoui, L., Feron, E. Balakrishnan, V. Linear Matrix Inequalities in System and Control Theory. SIAM Studies in Applied Mathematics, SIAM, 1994. https://doi.org/10.1137/1.97816119707777

8. Caponetto, R. Fractional Order Systems: Modeling and Control Applications. World Scientific, 2010. https:// doi.org/10.1142/978981430420r7

9. Caputo. M. Linear Models of Dissipation Whose Q is Almost Frequency Independent-II. Geophysical Journal International, 1967, 13(5), 529-539. https://doi. org/10.1111/j.1365-246X.1967.tb02303.x

10. Chen, W., Dai, H., Song, Y., Zhang, Z. Convex Lyapunov Functions for Stability Analysis of Fractional Order Systems. IET Control Theory \& Applications, 2017, 11(7), 1070-1074. https://doi.org/10.1049/iet-cta.2016.0950
11. Chen, M., Shao, S. Y., Shi, P., Shi, Y. Disturbance-Observer-Based Robust Synchronization Control for a Class of Fractional-Order Chaotic Systems. IEEE Transactions on Circuits and Systems II: Express Briefs, 2017, 64(4), 417-421. https://doi.org/10.1109/TCSII.2016.2563758

12. Chen, L. P., Wu, R. C. He, Y. G. Yin, L. Y. Robust Stability and Stabilization of Fractional-Order Linear Systems with Polytopic Uncertainties. Applied Mathematics and Computations, 2015, 257, 274-284. https://doi. org/10.1016/j.amc.2014.12.103

13. Chen, G., Yang, Y. Stability of a Class of Nonlinear Fractional Order Impulsive Switched Systems. Transactions of the Institute of Measurement and Control,2017,39(5), 781-790. https://doi.org/10.1177/0142331215621373

14. Cheng, J., Zhu, H., Zhong, S., Zheng, F., Zeng ,Y. Finite-Time Filtering for Switched Linear Systems with a Mode-Dependent Average Dwell Time. Nonlinear Analysis: Hybrid Systems, 2015, 15, 145-156. https://doi. org/10.1016/j.nahs.2014.09.001

15. Costa, O. L. V., Todorov, M. G., Fragoso, M. D. Continuous-Time Markov Jump Linear Systems. Springer-Verlag, Heidelberg, 2013. https://doi.org/10.10077/978-3642-34100-7

16. Faraji Niri, M. Robust Non-fragile Asynchronous Controller Design for Continuous-Time Markov Jump Linear Systems: Non-homogeneous Markov Process Approach. Circuits, Systems, and Signal Processing, 2018, 37(10), 4234-4255. https://doi.org/10.1007/s00034018-0767-y

17. Faraji Niri, M., Jahed Motlagh, M. R. Stochastic Stability and Stabilization of Semi-Markov Jump Linear Systems With Uncertain Transition Rates. Information Technology and Control, 2017, 46(1), 37-52. https://doi. org/10.5755/j01.itc.46.1.13881

18. Faraji Niri, M., Jahed Motlagh, M. R., Barkhordari Yazdi, M. Robust Stabilization of Uncertain Non-Homogeneous Markov Jump Linear Systems. 3rd International Conference on Control, Instrumentation, and Automation, Tehran, Tehran, 1 December 2013, 42-46. https:// doi.org/10.1109/ICCIAutom.2013.6912806

19. Faraji Niri, M., Jahed Motlagh, M. R., Barkhordari Yazdi, M. Stochastic Stability and Stabilization of a Class of Piecewise Homogeneous Markov Jump Linear Systems with Mixed Uncertainties. International Journal of Robust and Nonlinear Control, 2017, 27, 894-914. https://doi.org/10.1002/rnc.3602 
20. Flores Tlacuahuac, A., and Biegler, L. T. Optimization of Fractional Order Dynamic Chemical Processing Systems. Industrial and Engineering Chemistry Research, 2014, 53(13), 5110-5127. https://doi.org/10.1021/ ie $401317 \mathrm{r}$

21. Freeborn, T. J., Maundy, B., Elwakil, A. S. Fractional-Order Models of Supercapacitors, Batteries and Fuel Cells: a Survey. Materials for Renewable and Sustainable Energy, 2015, 4(3), 1-7. https://doi.org/10.1007/ s40243-015-0052-y

22. HosseinNia, S. H., Tejado, I., Vinagre, B. M. Stability of Fractional Order Switching Systems. Computers and Mathematics with Applications. 2013, 66, (5), 585-596. https://doi.org/10.1016/j.camwa.2013.05.005

23. Khooban, M. H., Niknam, T., Shasadeghi, M., Dragicevic, T., Blaabjerg, F., Load Frequency Control in Microgrids Based on a Stochastic Non-integer Controller. IEEE Transactions on Sustainable Energy, 2018, 9(2), 853-861. https://doi.org/10.1109/TSTE.2017.2763607

24. Khooban, M. H., ShaSadeghi, M., Niknam, T., Blaabjerg, F., Analysis, Control and Design of Speed Control of Electric Vehicles Delayed Model: Multi-Objective Fuzzy Fractional-Order PID Controller. IET Science, Measurement \& Technology, 2016, 11(3), 249-61. https://doi.org/10.1049/iet-smt.2016.02777

25. Li, C. P., Zhang, F. R. A Survey on The Stability of Fractional Differential Equations. The European Physical Journal-Special Topics, 2011, 193(1), 27-47. https://doi. org/10.1140/epjst/e2011-01379-1

26. Liang, S., Wu, R. C., Chen, L. P. BIBO Stability of Fractional-Order Controlled Nonlinear Systems, International Journal of System Sciences, 2017, 48(7), 15071514. https://doi.org/10.1080/00207721.2016.1269219

27. Lin, H., Antsaklis P. J. Stability and Stabilizability of Switched Linear Systems: a Survey of Recent Results. IEEE Transactions on Automatic control, 2009, 54(2), 308-322. https://doi.org/10.1109/TAC.2008.2012009

28. Liu L., CaoX., FuZ., SongS. Guaranteed CostFinite-Time Control of Fractional-Order Positive Switched Systems. Advances in Mathematical Physics, 2017, 32(3), 857-874, https://doi.org/10.1155/2017/2695894

29. Liu, R. J., Nie, Z. Y., Wu, M., She, J. Robust Disturbance Rejection for Uncertain Fractional-Order Systems. Applied Mathematics and Computation, 2018, 322, 79-88. https://doi.org/10.1016/j.amc.2017.11.021

30. Long, F., Fei, S., Fu, Z., Zheng, S., Wei, W. Hœ Control and Quadratic Stabilization of Switched Linear Systems with Linear Fractional Uncertainties via Output
Feedbac. Nonlinear Analysis: Hybrid Systems, 2008, 2, 18-27. https://doi.org/10.1016/j.nahs.2006.11.004

31. Monje, A., Chen, Y. Q., Vinagre, B. M., Xue, D., Feliu, V. Fractional-Order Systems and Controls. Fundamentals and Applications, Springer-Verlag, London, 2010. https://doi.org/10.1007/978-1-84996-335-0

32. Moze, M., Sabatier, J., Oustaloup, A. LMI Characterization of Fractional Systems Stability. Advances in Fractional Calculus, 2007, 419-434. https://doi. org/10.1007/978-1-4020-6042-7_29

33. Niu, B., Zhao, J. Tracking Control for Output-Constrained Nonlinear Switched Systems with a Barrier Lyapunov Function. International Journal of Systems Science. 2013, 44(5), 978-985. https://doi.org/10.1080/ 00207721.2011.652222

34. Poursafar, N., Taghirad, H.D., Haeri, M., Model Predictive Control of Non-Linear Discrete Time Systems: a Linear Matrix Inequality Approach. IET Control Theory \& Applications, 2010, 4(10), 1922-1932. https://doi. org/10.1049/iet-cta.2009.0650

35. Rajagopal, K., Vaidyanathan, S., Karthikeyan, A., Duraisamy, P. Dynamic Analysis and Chaos Suppression in a Fractional Order Brushless DC Motor. Electrical Engineering, 2017, 99(2), 721-733. https://doi.org/10.1007/ s00202-016-0444-8

36. Sabatier, J., Mathieu, M., Christophe, F. LMI Stability Conditions for Fractional Order Systems, Computers \& Mathematics with Applications, 2010, 59(5), 15941609. https://doi.org/10.1016/j.camwa.2009.08.003

37. Sun, Z. Switched Linear Systems: Control and Design. Springer Science and Business Media, 2006.

38. Sun, Z., Ge, S. S. Stability Theory of Switched Dynamical Systems. Springer Science \& Business Media, 2011. https://doi.org/10.1007/978-0-85729-256-8

39. Tavazoei, M. S., Haeri, M. A Note on the Stability of Fractional Order Systems, Mathematics and Computers in Simulation, 2009, 79(5), 1566-1576 https://doi. org/10.1016/j.matcom.2008.07.003

40. Trigeassou, J. C., Maamri, N., Sabatier, J., Oustaloup, A. A Lyapunov Approach to the Stability of Fractional Differential Equations. Signal Processing, 2011, 91, 437445. https://doi.org/10.1016/j.sigpro.2010.04.024

41. Wang, R., Xing, J., Xiang, Z. Finite-Time Stability and Stabilization of Switched Nonlinear Systems with Asynchronous Switching. Applied Mathematics and Computation, 2018, 316, 229-244. https://doi. org/10.1016/j.amc.2017.08.017 
42. Xie, L. H. Output Feedback $\mathrm{H} \infty$ Control of Systems with Parameter Uncertainty. International Journal of Control, 1996, 63(4), 741-750. https://doi. org/10.1080/00207179608921866

43. Xu, B. B., Chen, D. Y., Zhang, H., Wang, F. F. Modeling and Stability Analysis of a Fractional-Order Francis Hydro-Turbine Governing System. Chaos, Solitons and Fractals, 2015, 75, 50-61. https://doi.org/10.1016/j.chaos.2015.01.025

44. Yang, Y., Chen, G. Finite-Time Stability of Fractional Order Impulsive Switched Systems. International Journal of Robust and Nonlinear Control, 2015, 25(13), 2207-2222. https://doi.org/10.1002/rnc.3202

45. Yang, J., Chen, Y, Zhu, F., Yu, K., Bu, X. Synchronous Switching Observer for Nonlinear Switched Systems with Minimum Dwell Time Constraint. Journal of Franklin Institute, 2015, 352(11), 4665-4681. https:// doi.org/10.1016/j.jfranklin.2015.07.010
46. Yang, H., Jiang B. Stability of Fractional-Order Switched Non-Linear Systems. IET Control Theory \& Applications, 2016, 10(8), 965-970. https://doi.org/10.1049/ietcta.2015.0989

47. Zhang, L., Songlin, Z., Shi, P. Non-Weighted Quasi-Time-Dependent $\mathrm{H} \infty$ Filtering for Switched Linear Systems with Persistent Dwell-Time. Automatica, 2015, 54, 201-209. https://doi.org/10.1016/j.automatica.2015.02.010

48. Zhao, X., Yin, Y., Zheng, X. State-Dependent Switching Control of Switched Positive Fractional-Order Systems. ISA Transactions, 2016, 62, 103-108. https://doi. org/10.1016/j.isatra.2016.01.011

49. Zhao, X., Zheng, X., Niu, B., Liu, L. Adaptive Tracking Control for a Class of Uncertain Switched Nonlinear Systems. Automatica. 2015, 52, 185-191. https://doi. org/10.1016/j.automatica.2014.11.019 